\title{
CMV in Moderately Active Colitis: Much Ado About Nothing?
}

\author{
Alan C. Moss
}

Published online: 4 March 2010

(C) Springer Science+Business Media, LLC 2010

Cytomegalovirus (CMV) infection is prevalent in $\sim 70 \%$ of patients with inflammatory bowel disease (IBD), but whether this virus contributes to disease severity or adverse outcomes has long been a matter of debate [1,2]. After initial infection, CMV enters a latent phase that has no clinical consequences, unless the virus is re-activated in the colon during periods of physiological stress or immunosuppression. If CMV is re-activated, local cytopathic effects in the colon can lead to a symptomatic colitis (CMV disease) that is very similar to IBD. In patients with severe and/or steroid-refractory colitis, there is evidence that such colonic CMV re-activation occurs in approximately $30 \%$ of cases [3]. Clinical guidelines have recommended that we test for colonic CMV disease in patients with severe colitis, and treat CMV+ patients with antiviral therapy $[4,5]$.

The key question that arises in these patients is whether the CMV re-activation has actually worsened the colitis, or is merely a by-product of escalating inflammation. It is known from laboratory studies that local re-activation of latent CMV can occur due to macrophage transformation, and the presence of TNF, IFN- $\gamma$, and hydrocortisone; all consequences of severe colitis [6, 7]. If CMV re-activation adversely affects clinical outcomes, then antiviral treatment should induce remission, and prevent these patients from going to colectomy. While some studies have reported clinical improvement in patients with colitis and colonic CMV who were treated with ganciclovir, this was often in combination with immunosuppressive therapy for colitis $[3,8]$. The small numbers reported makes definitive

\footnotetext{
A. C. Moss ( $\square)$

Harvard Medical School, 330 Brookline Ave, Boston, MA 02215, USA

e-mail: amoss@bidmc.harvard.edu
}

conclusions about the impact of antiviral therapy difficult to quantify.

The existing literature has been dominated by a number of confounding issues that have maintained the uncertainties of this topic [9];

1. the prevalence studies are mostly in patients with severe colitis who are treated with steroids

2. the methods to define colonic CMV disease have been inconsistent across studies

3. there is little data on the outcomes of patients with CMV disease who did not receive antiviral therapy

In order to clarify the impact of CMV re-activation in colitis, additional studies on the prevalence of CMV disease in patients with mild-moderate disease, and those taking steroids that don't have severe disease, are lacking. In addition, natural-history data on patients with colitis who had untreated CMV detected in their colon are scarce.

The paper by Kim et al. in the current edition of this journal addresses some of these issues [10]. In this retrospective archival study, the authors identified the biopsy specimens of 142 patients with known or suspected IBD who underwent a sigmoidoscopy or colonoscopy for symptoms of active colitis. All specimens were re-examined by $\mathrm{H} \& \mathrm{E}$ staining, and each of the patients' most inflamed specimens were also stained for CMV immediate early antigen by IHC. Approximately half $(48 \%)$ of the patients were newly diagnosed with IBD, and half $(43 \%)$ were hospitalized at the time of the procedure. Of the 122 patients with ulcerative colitis (UC), only $26 \%$ were being treated with an immunosuppressant (steroids, immunomodulators, biologic).

Among the 122 patients with UC, none had typical viral disease features on H\&E stain, but $12(10 \%)$ had positive IHC staining for CMV. The prevalence of colonic CMV 
was low in patients with moderate disease $(2 \%$ in those who did not require hospitalization or steroids, and $4 \%$ in patients who did not require hospitalization). When the 12 cases with CMV were examined, about half $(42 \%)$ had a new diagnosis of IBD, and were on no treatment for IBD, and a similar proportion (58\%) did not require hospitalization for their colitis. Proportionally, patients with CMV were more likely to have a WCC $>10,300 / \mathrm{mm}^{3}$, and require hospitalization for $>7$ days, when compared to CMV- patients. Where follow-up data was available, 5/10 $\mathrm{CMV}+$ patients had a further hospitalization for their colitis compared to 20/82 CMV - patients.

Interestingly, none of the CMV+ patients were diagnosed with colonic CMV during their initial flare, and 9/12 (75\%) were subsequently treated with steroids. Of these, $6 / 9(67 \%)$ had no further flares requiring hospitalization on follow-up. The only patient diagnosed with $\mathrm{CMV}$ and treated with antivirals eventually failed infliximab and underwent a colectomy.

There are a couple of useful messages from this study. Firstly, patients who indirectly appeared to have had moderate disease (no requirement for steroids or hospitalization, but warranted a sigmoidoscopy) had a low prevalence of CMV in their colon (2\%). This is consistent with other studies that included patients with mild to moderate colitis, where CMV prevalence by IHC in endoscopic biopsies was $0 \%[3,11]$. The absence of CMV in the patients with Crohn's colitis also confirms other data in this group of patients $[12,13]$.

Secondly, patients with colonic CMV who were subsequently treated with steroids, and not antivirals, did not have worse outcomes on follow-up. Only $3 / 9$ (33\%) had a further flare requiring hospitalization, which was similar to the rate (24\%) amongst the 82 patients without CMV in their colon. Since the univariate comparison between $\mathrm{CMV}+$ and $\mathrm{CMV}-$ patients did not control for disease severity, it cannot be determined whether the higher WCC, hospital stay, and hospitalization rate in the $\mathrm{CMV}+$ group is due to CMV disease, or to more severe colitis. The small number (12) of patients with CMV would not allow meaningful sub-group comparisons for this purpose.

This study complements the existing literature on CMV in IBD by providing prevalence data in patients with mild to moderate active disease, in contrast to the many studies that looked for CMV in colectomy specimens in steroidrefractory patients. Most studies of steroid-refractory patients have detected CMV by IHC in $30-60 \%$ of patients who had a colectomy $[14,15]$. By comparison, Domenech et al. detected no CMV by IHC in 25 patients with active UC that required IV steroids, or 50 patients with inactive $\mathrm{UC}$, even in those taking immunomodulators [3]. The authors have also provided further evidence that missing, or not treating, colonic CMV in this population does not adversely affect patient outcomes. Matsuoka et al. reported outcomes in patients with moderate to severe colitis who had CMV reactivation by PCR in their blood, but were not treated with antivirals [16]. Patient outcomes were similar between CMV + and CMV - patients in this study. Yoshino et al. also reported that the finding of CMV in the colon of steroid-refractory patients with UC was not associated with disease activity or colectomy rates in a small study [17]. Testing whether colonic CMV disease influences patient outcomes in sicker patients is fraught with difficulties, where the fear of not treating detected CMV outweighs unnecessary treatment with antivirals in the setting of severe colitis. Previous studies in this patient population have contained a mixed bag of those who were treated with antivirals and immunosuppressants, and those where only antivirals were used $[3,8,17,18]$.

The limitations to this study include the retrospective nature of the data capture and the fact that no direct measurement of patients' severity was provided, either by clinical or endoscopic scores. The association between CMV status and variables such as WCC and hospital stay may therefore be confounded by disease severity. Using hospitalizations as an indirect measure of disease activity, will miss less severe disease flares that may have differed between CMV+ and CMV - groups. Overall, however, the authors are to be commended for enhancing the existing data on this topic.

In summary, this study demonstrates that reactivation of CMV in patients with moderately active colitis is uncommon, and does not adversely impact patient outcomes when untreated (Table 1). This strengthens the case that colonic CMV reactivation is predominantly a by-stander in exacerbations of colitis, rather than the factor leading to disease flares. In patients with severe, steroid-refractory disease, this issue is more difficult to clarify. It would require either a large multi-center cohort study to compare remission and colectomy rates in $\mathrm{CMV}+$ and $\mathrm{CMV}-$ patients with comparable disease activity, or a controlled trial to compare standard treatment with or without ganciclovir in those with colonic CMV. In the absence of this evidence,

Table 1 Summary of key points

Colonic CMV reactivation is rare in patients with mild to moderate ulcerative colitis and colonic Crohn's disease

Untreated colonic CMV reactivation does not appear to adversely affect patient outcomes in this patient population

The impact of colonic CMV in patients with severe/steroid-refractory colitis is unclear

Patients with severe/steroid-refractory colitis, who are infected with CMV (by serology), should be tested for colonic CMV reactivation (by tissue IHC or blood PCR) and treated if positive

The evidence to support antiviral therapy in this setting is currently based on a small case series only 
one approach is to test for CMV reactivation (via blood DNA PCR or tissue IHC) in patients with severe steroidrefractory colitis who are infected with CMV (serum CMV $\mathrm{IgG}+$ ). Patients with evidence of re-activation should have ganciclovir added to their existing therapy, unless systemic CMV disease requires discontinuation of immunosuppressants. The input of an infectious disease and surgical colleague are essential in this setting.

\section{References}

1. Lawlor G, Moss AC. Cytomegalovirus in inflammatory bowel disease; pathogen or innocent bystander? Inflamm Bowel Dis. 2010 (in press).

2. Hommes DW, Sterringa G, van Deventer SJ, Tytgat GN, Weel J. The pathogenicity of cytomegalovirus in inflammatory bowel disease: a systematic review and evidence-based recommendations for future research. Inflamm Bowel Dis. 2004;10:245-250.

3. Domenech E, Vega R, Ojanguren I, et al. Cytomegalovirus infection in ulcerative colitis: a prospective, comparative study on prevalence and diagnostic strategy. Inflamm Bowel Dis. 2008; 14:1373-1379.

4. Kornbluth A, Sachar DB. Ulcerative colitis practice guidelines in adults (update): American College of Gastroenterology, Practice Parameters Committee. Am J Gastroenterol. 2004;99:1371-1385.

5. Rahier JF, Yazdanpanah Y, Colombel JF, Travis S. The European (ECCO) consensus on infection in IBD: what does it change for the clinician? Gut. 2009;58:1313-1315.

6. Soderberg-Naucler C, Fish KN, Nelson JA. Interferon-gamma and tumor necrosis factor-alpha specifically induce formation of cytomegalovirus-permissive monocyte-derived macrophages that are refractory to the antiviral activity of these cytokines. J Clin Invest. 1997;100:3154-3163.

7. Widmann T, Sester U, Gartner BC, et al. Levels of CMV specific CD4 $\mathrm{T}$ cells are dynamic and correlate with CMV viremia after allogeneic stem cell transplantation. PLoS One. 2008;3:e3634.
8. Papadakis KA, Tung JK, Binder SW, et al. Outcome of cytomegalovirus infections in patients with inflammatory bowel disease. Am J Gastroenterol. 2001;96:2137-2142.

9. Ayre K, Warren BF, Jeffrey K, Travis SPL. The role of CMV in steroid-resistant ulcerative colitis: a systematic review. J Crohn's Colitis. 2009 (in press).

10. Kim JJ, Simpson N, Klipfel N, DeBose R, Barr N, Laine L. Cytomegalovirus infection in patients with active inflammatory bowel disease. Dig Dis Sci. 2010. doi:10.1007/s10620-0101126-4.

11. D’Ovidio V, Vernia P, Gentile G, et al. Cytomegalovirus infection in inflammatory bowel disease patients undergoing antiTNFalpha therapy. J Clin Virol. 2008;43:180-183.

12. Kaufman HS, Kahn AC, Iacobuzio-Donahue C, Talamini MA, Lillemoe KD, Hamilton SR. Cytomegaloviral enterocolitis: clinical associations and outcome. Dis Colon Rectum. 1999;42: 24-30.

13. Knosel T, Schewe C, Petersen N, Dietel M, Petersen I. Prevalence of infectious pathogens in Crohn's disease. Pathol Res Pract. 2009;205:223-230.

14. Kambham N, Vij R, Cartwright CA, Longacre T. Cytomegalovirus infection in steroid-refractory ulcerative colitis: a casecontrol study. Am J Surg Pathol. 2004;28:365-373.

15. Kuwabara A, Okamoto H, Suda T, Ajioka Y, Hatakeyama K. Clinicopathologic characteristics of clinically relevant cytomegalovirus infection in inflammatory bowel disease. $J$ Gastroenterol. 2007;42:823-829.

16. Matsuoka K, Iwao Y, Mori T, et al. Cytomegalovirus is frequently reactivated and disappears without antiviral agents in ulcerative colitis patients. Am J Gastroenterol. 2007;102: 331-337.

17. Yoshino $T$, Nakase $H$, Ueno $S$, et al. Usefulness of quantitative real-time PCR assay for early detection of cytomegalovirus infection in patients with ulcerative colitis refractory to immunosuppressive therapies. Inflamm Bowel Dis. 2007;13:15161521.

18. Cottone M, Pietrosi G, Martorana G, et al. Prevalence of cytomegalovirus infection in severe refractory ulcerative and Crohn's colitis. Am J Gastroenterol. 2001;96:773-775. 